\title{
The non-invasive molecular method of biocybernetic dermovisceral clavitherapy, non invasive
}

\section{Introduction}

Dear Friend, according to American research in a healthy man's blood there are 3 million various types of molecular endogenous factors, including approximately 100thousand selective antibodies (my own finding). Furthermore, in blood there are approximately 10 million submolecular neurochemical transmitters and neurotransmitters. All the molecular factors, antibodies and submolecular neurochemical transmitters are subject to biocybernetical guidance on a noninvasive basis through skin receptors, nerved collagen fibres, multiple biologically active points, including cryogenic, acupunctural ones and the nervous system. I have methodically embraced the whole method of clavitherapy, which I have been researching and experimenting on for over 50years with verification in clinical condition, in 1100 algorithms with the network of activities quantified with medical diagnosis in the books: Klawiterapia [Clavitherapy] and Atlas klawiterapii [Atlas of clavitherapy]. In general terms the art of clavitherapy should be assumed as the art of stimulation with clavicles similar to nails (Latin Clavus=nail) in dermo-visceral guidance of corrective processes of the homeostasis of the human organism, impaired molecularly from negligence and ischemia. The second clinical achievement and even my discovery was precise etiological determination of functional and structural disorders of the nervous system constituting the main causes of many medication resistant disorders. In the 1980s at the Neurologic Clinic of the Clinical Hospital of the Ministry of Interior in Warsaw I developed an effective simple short-term method of liquidation of demyelinised disorders in the brain and in various types of peripheral nerve fibres and neuron palsy with gliomas caused by the earliest changes of malignant tumours, which are also medication resistant. The scope of the effectiveness of the clavitherapy method is expressed in summary of the enclosed publication entitled Medycyna molekularna odległej przyszłości dostępna już dzisiaj [Molecular medicine of distant future available as early as today]. It also concerns resuscitation and reanimation without a defibrillator and chest massage. At present there are already a few masters of clavitherapy and two doctoral dissertations have been pursued at universities of medicine. The fourth edition of Clavitherapy has been prepared but I have no funds to have it printed. In anticipation of successful implementation of the method of clavitherapy in didactic,

\author{
Volume 2 Issue 3 - 2016
}

Ferdynand Barbasiewicz

The creator of the method, University of warsaw, Poland

Correspondence: Ferdynand Barbasiewicz, The creator of the method, University of warsaw, Poland, Tel +48 227298053 , Email centrum@klawiterapia.com

Received: May 31, 2016 | Published: June 14, 2016

research and specialisation programs at medical institutions of higher education, I would like you to acquaint yourselves with the position of the Polish Medical Association. Please, read the enclosed publications and watch the links of short films: ATVN on www.klawiterapia.com and address of the Polish Medical Association authorities on www. youtabe.com/wath? $\mathrm{v}=\mathrm{ymZOfeYLDtk}$ www.instytutklawiterapii.com/ yt Let me also inform you that at Centrum Klawiterapii - Źródło [the Centre of Clavitherapy - Source] I am in possession of many documents constituting clinical evidence of my activities. Moreover, enter the English term Clavitherapy in Google to see how the method of clavitherapy is propagated all over the world. You will find more information on the above mentioned websites.

I wish you all the best and a lot of success in provision of medical services.

Respectfully yours, Ferdynand Barbasiewicz, the creator of the method of clavitherapy and author of e-book: EDEN XXI WIEKU [EDEN OF THE $21^{\mathrm{ST}}$ CENTURY] (regarding objective, technically simple possibilities of reconstruction of man's ecological negligence).

\section{Acknowledgements}

None.

\section{Conflict of interest}

The author declares no conflict of interest. 